\title{
SETTING UP AN ENHANCED SURVEILLANCE OF NEWLY ACQUIRED HEPATITIS C INFECTION IN MEN WHO HAVE SEX WITH MEN: A PILOT IN LONDON AND SOUTH EAST REgION OF ENGLAND
}

\author{
M Ruf (murad.ruf@hpa.org.uk) ${ }^{1}$, S Cohuet ${ }^{2,3}$, H Maguire ${ }^{2}$, L J Brant ${ }^{1}$, M Ramsay ${ }^{1}$, S Lattimore ${ }^{1}$, V Delpech ${ }^{1}$, the SNAHC steering \\ group 4 \\ 1. Centre for Infections, Health Protection Agency, England and Wales \\ 2. London Region Epidemiology Unit, Health Protection Agency, England and Wales \\ 3. European Programme for Intervention Epidemiology Training (EPIET), Stockholm, Sweden \\ 4. The members are listed in the Acknowledgements section
}

We describe the implementation of an enhanced surveillance pilot for newly acquired hepatitis $\mathrm{C}(\mathrm{HCV})$ in men who have sex with men (MSM) in London and the South East region of England. Preliminary findings suggest ongoing HCV transmission among MSM infected with human immunodeficiency virus (HIV) and that enhanced surveillance for newly acquired HCV in MSM is feasible.

\section{Background}

Acute hepatitis $\mathrm{C}(\mathrm{HCV})$ among people living with human immunodeficiency virus (HIV) is increasingly being recognised as an emerging problem in developed countries following the wide introduction of anti-retroviral therapy (ART) [1]. In recent years there has been a rise in reports of newly acquired HCV infection among HIV-infected men who have sex with men (MSM) without a history of injecting drug use (IDU). Mucosal trauma during highrisk sexual practices has been suggested as the most likely route of transmission among MSM [2-5].

In 2006, a retrospective study estimated an average annual rise of $20 \%$ in the incidence of newly diagnosed HCV among HIVpositive MSM attending genito-urinary medicine (GUM) clinics in London and Brighton since 2002. 389 cases were reported among HIV-positive MSM, but only six cases were reported in HIV-uninfected MSM for the four and a half year period. [6] The findings reinforced the need for routine and repeat HCV testing of HIV-infected individuals, in line with guidelines of the British HIV Association (BHIVA) [7].

To better understand the burden of HCV infection and associated behavioural risk factors among MSM, the Health Protection Agency (HPA), in collaboration with BHIVA and the British Association for Sexual Health and HIV (BASSH), is piloting enhanced surveillance of newly acquired HCV in MSM (SNAHC) in London and South East region of England. We describe the set-up of the SNAHC pilot and report preliminary findings for the period from 1 January to 30 September 2008.

\section{Methods}

The SNAHC project was developed under the guidance of a multidisciplinary steering group [8]. In February 2008, all 35 GUM clinics in London and five major clinics in the South East region of England were invited to participate by email and a postal letter. The pilot was also announced at the annual BHIVA spring conference in 2008. Full project information was made available on a dedicated page on the HPA website (see link at the end of the article). Starting from January 2008, participating clinics were required to report all cases of newly acquired HCV in MSM using a standard reporting form requesting the following: HCV antibody and RNA test results, HCV genotype, reason for HCV testing, HIV status and date of HIV diagnosis, most recent CD4 count, ART, history of IDU, sexually transmitted infections (STI), sexual practices and recreational drug use. Monthly reminder emails to report new cases are sent to identified clinic contacts.

The surveillance case definitions for newly acquired HCV are:

- Confirmed case: HCV-antibody sero-conversion within the previous 36 months;

- Probable case: HCV-RNA-positive and HCV-antibody-negative or equivocal.

In order to close the surveillance gap between the previous study in mid-2006 and the start of SNAHC, clinics were asked to retrospectively report the aggregate number of cases seen in 2006 and 2007. HCV incidence estimates for HIV-positive MSM were calculated using as denominator person years (PY) of MSM with diagnosed HIV infection attending care, consistent with the study by Giraudon et al. [6].

\section{Results}

Sixty percent (25/40) of invited clinics in the targeted regions agreed to participate, including all major HIV treatment centres, covering $91 \%$ of MSM attending for HIV care in London and $57 \%$ in the South East region [8]. 


\section{Retrospectively reported HCV cases in HIV-infected MSM 2006 and 2007}

A total of 200 cases in HIV-infected MSM were reported retrospectively from all participating clinics for 2006 and 2007 (91 cases in 2006, 109 in 2007).

\section{HCV cases and estimated incidence in diagnosed HIV-infected MSM 2002-2007}

For the sixteen clinics that had reported cases since 2002 [6], the annual cases and the estimated annual incidence for diagnosed HIV-infected MSM in the period from 2006 to 2007 has shown no obvious trend, with 84 cases in 2006 (incidence: 7.4/1,000 PY) and 101 cases in 2007 (8.2 per 1,000 PY) (Figure). In 2007, these sixteen clinics provided care for $85 \%$ of diagnosed HIVinfected MSM in London and 56\% in Brighton.

Case numbers reported for 2006 and 2007 (nil cases) from one large London clinic included in the Figure were inconsistent and are being further investigated. The case numbers and incidence for 2006 and 2007 are therefore probably underestimates of the true figures. Despite this missing information the number of reported cases has remained high.

\section{Prospectively reported cases since January 2008}

In the first nine months of the pilot, a total of 29 cases were reported from 11 of the 25 participating clinics. This is less than $40 \%$ of the number of cases expected for this period based on numbers reported retrospectively in the same clinics during 2006 and 2007. The median lead time between serological diagnosis and completed report was around two months. Ten forms were insufficiently completed, not allowing classification as confirmed

\section{F I G U R E}

Numbers of cases and estimated incidence of newly acquired HCV in diagnosed HIV-infected MSM reported by HIV clinics in London and Brighton, 2002-2007

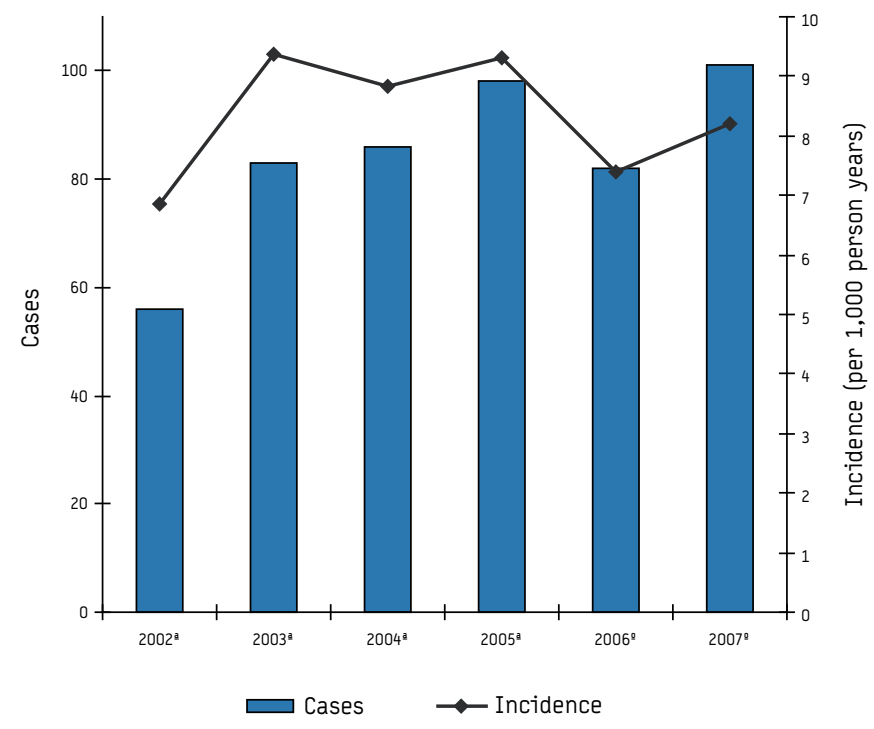

or probable cases. After follow-up of missing information and strict application of the case definition the steering group verified 17 as confirmed and 12 as probable cases. All but one man had a known HIV-positive status at the time of HCV infection. The sections on behavioural risk factors were well completed. IDU was denied by all but three cases (two of which reported use within the last three months, one reported last use eight years previously); $72 \%$ of cases $(21 / 29)$ gave information regarding fisting practices, $57 \%$ of those $(12 / 21)$ denied any fisting in the previous three months.

\section{Discussion}

The pilot shows the feasibility of enhanced surveillance of newly acquired HCV in MSM. The preliminary findings suggest ongoing HCV transmission among HIV-infected MSM in London and the South East region of England. The clinics that agreed to participate in the surveillance scheme provide care for the majority of diagnosed HIV-infected MSM in those regions and should therefore be representative of the population of interest. The low number of reported cases in HIV-uninfected MSM is consistent with previous studies [6]. However, the surveillance may be biased towards HIV-infected MSM, as regular HCV testing is recommended for individuals with diagnosed HIV infection in the United Kingdom while no such recommendation currently exists for other MSM.

Incompleteness of information and completion errors highlighted design weaknesses of the case definition section of the reporting form; the form is now being amended. Secondly, fewer reports than expected have been received for the period compared to the numbers reported in previous years. Apart from a genuine fall in incidence, likely reasons include under-reporting and reporting delay due to the need to complete laboratory, clinical and behavioural information. To address the latter issue, the steering group has suggested asking clinicians to report new cases at the time of the initial diagnosis, with subsequent follow-up for behavioural information and additional serology.

A comprehensive report will be prepared at the end of the 12 months pilot phase of SNAHC, allowing sufficient time for possible reporting delays. Preliminary results suggest that SNAHC will be able to provide useful information for the epidemiology and control of newly acquired HCV in MSM.

With around 100,000 MSM aged 16-44 years and nearly 12,000 HIV-positive MSM accessing care in 2007 [8,9], London has a substantial MSM population at risk. The city also constitutes a hub for national and international sexual and infection networks of MSM. We welcome knowledge of similar surveillance schemes for newly acquired HCV in MSM in other European countries. Further information on SNAHC, protocol and reporting forms are available at: http://www.hpa.org.uk/web/HPAweb\&HPAwebStandard/ HPAweb_C/1204186211416 or under www.hpa.org.uk, keyword 'SNAHC'.

Acknowledgements:

The SNAHC steering group consists of the authors plus: Martin Fisher, Anna-Maria Geretti, Sanjay Bhagani, David Asboe, Jackie Cassell, Paul Crook, Rohini Manuel, Grainne Nixon and Angela Iversen 


\section{References}

1. Danta M, Dusheiko GM. Acute HCV in HIV-positive individuals - a review. Curr Pharm Des. 2008;14(17):1690-7.

2. Browne R, Asboe D, Gilleece Y, Atkins M, Mandalia S, Gazzard B, et al. Increased numbers of acute hepatitis $C$ infections in HIV positive homosexual men; is sexual transmission feeding the increase? Sex Transm Infect. 2004;80(4):326-7.

3. Götz HM, van Doornum G, Niesters HG, den Hollander JG, Thio HB, de Zwart 0 , et al. A cluster of acute hepatitis $C$ virus infection among men who have sex with men-results from contact tracing and public health implications. AIDS 2005;19(9):969-74.

4. Danta M, Brown D, Bhagani S, Pybus OG, Sabin CA, Nelson M, et al. Recent epidemic of acute hepatitis $C$ virus in HIV-positive men who have sex with men linked to high-risk sexual behaviours. AIDS 2007;21(8):983-91.

5. Gilleece YC, Browne RE, Asboe D, Atkins M, Mandalia S, Bower M, et.al Transmission of hepatitis $C$ virus among HIV-positive homosexual men and response to a 24-week course of pegylated interferon and ribavirin. J Acquir Immune Defic Syndr. 2005;40(1):41-6.

6. Giraudon I, Ruf M, Maguire H, Charlett A, Ncube F, Turner J, et al. Increase in diagnosed newly acquired hepatitis $C$ in HIV-positive men who have sex with men across London and Brighton, 2002-2006: is this an outbreak? Sex Transm Infect. 2008;84(2):111-5.

7. British HIV Association. British HIV Association (BHIVA) guidelines for treatment and management of HIV and Hepatitis C coinfection. London: BHIVA; 2004 Oct. Available from: http://www.bhiva.org/files/file1001579.pdf

8. Johnson AM, Mercer CH, Erens B, Copas AJ, McManus S, Wellings K, et al. Sexual behaviour in Britain: partnerships, practices, and HIV risk behaviours. Lancet. 2001;358(9296):1835-42.

9. HIV/STI Division, Health Protection Agency, Centre For Infections. Survey of Prevalent HIV Infections Diagnosed (SOPHID) [homepage on the internet] London: Health Protection Agency. Available from: http://www.hpa.org.uk/webw/ HPAweb\&Page\&HPAwebAutoListDate/Page/1201094588844? $p=1201094588844$

This article was published on 20 November 2008.

Citation style for this article: Ruf M, Cohuet S, Maguire H, Brant LJ, Ramsay M, Lattimore $S$, Delpech V, the SNAHC steering group. Setting up an enhanced surveillance of newly acquired hepatitis $C$ infection in men who have sex with men: a pilot in London and South East region of England. Euro Surveill. 2008;13(47):pii=19042. Available online: http://www.eurosurveillance.org/ViewArticle.aspx?ArticleId=19042 